T. SHrmrzu, Independent sets and measure algebras ....... 209-225 L. WAELBROECK, A rigid topological vector space . . . . . . . . . $227-234$ D. M. OBERLtN, Multipliers of $L_{R}^{p}$, II . . . . . . . . . . . 235-248 T. BrCzkowskr, Gaussian measures on $L_{p}$ spaces, $0 \leqslant p<\infty \ldots 249-261$ W. WoJTYর́SKI, Banach-Lie algebras of compact operators . . . . . . 263-273 K. URBANIK, A characterization of Gaussian measures on Banach spaces 275-281 E. Dubinskr, Basic sequences in $(s)$. . . . . . . . . . . . . 283-293 V. WroBex, Beschränkte Oporatoren: Bemerkungen zu einer Arboit von P. Uss . . . . . . . . . . . . . . . . . . . 295-300 K.-H. FöRSTER and E. - O. LIEBETRAU, On semi-Fredholm operators and the

conjugate of a product of operators . . . . . . . . 301-306

STUDIA MATHEMATICA

Managing editors: Z. Giesielski, W. Orlicz (Editor-in-Ohief), A. Pelczyński, W. Żelazko

The journal prints original papers in English, French, German and Russian, mainly on functional analysis, abstract methods of mathematical analysis and on the theory of probabilities. Usually 3 issues constitute a volume.

The papers submitted should be typed on one side only and they should be accompanied by abstracts, normally not exceeding 200 words in length. The authors are requested to send two copies, one of them boing the typed, not Xerox copy. Authors are advised to retain a copy of the paper submitted for publication.

Manuscripts and the correspondence concerning the editorial work should be addressed to

\section{TUDIA MATHEMATICA}

ul. Śniadeckich 8

00-950 Warszawa, Poland

Correspondence concerning exchange should be addressed to

INSTITUTE OF MATHEMATICS

POLISH ACADEMY OF SCIENCES

ul. Sniadeckich 8

00-950 Warszawa, Poland

The journal is available at your booksellor or at

$$
\text { ARS POLONA }
$$

Krakowskie Przedmieścio 7

00-068 Warszawa, Poland

PRINTED IN POLAND

\section{Independent sets and measure algebras}

by $\quad$ \&y

TETSUHIRO SHIMIZU (Sapporo, Hokkaido)

Abstract. Let $G$ be a non-discrete locally compact abelian group. Let $M(G)$ be the measure algebra on $G$. In this paper, at first, we shall consider the relation between independent sets and prime $L$-subalgebras of $M(G)$. Finally, we shall show the existence of measures with some properties in case of $G$ being compact and the dual group of $G$ having an infinite independent set.

o. Introduction. Throughout this paper $G\left(\tau_{0}\right)$ denotes a non-discrete locally compact abelian group with an underlying group $G$ and a topology $\tau_{0}$. We shall denote by $\mathscr{T}\left(G\left(\tau_{0}\right)\right)$ the set of all those locally compact group topologies on $G$ which are stronger than or equal to the original topology $\tau_{0}$. For any $\tau \in \mathscr{T}\left(G\left(\tau_{0}\right)\right)$, let $M(G(\tau))$ be the algebra of all bounded regular Borel measures on $G(\tau)$ under convolution multiplication, and $L^{1}(G(\tau))$ the ideal consisting of all those measures which are absolutely continuous with respect to the Haar measure $m_{\tau}$ on $G(\tau)$.

A closed subalgebra $N$ of $M\left(G\left(\tau_{0}\right)\right)$ is called an $L$-subalgebra if $\mu \epsilon N$ and $\nu \ll \mu$ implies $\nu \in N$. An $L$-subalgebra $N$ is said to be prime if $N^{\perp}$ is an ideal, where $N^{\perp}$ is the set of measures $\nu$ such that $\nu \pm \mu$ for all $\mu \epsilon N$.

A collection $\mathscr{F}$ of $\sigma$-compact subsets of $G\left(\tau_{0}\right)$ is called a Raikov system if the following conditions are satisfied:

(R1) If $A_{1} \in \mathscr{F}$ and $A_{2}$ is a $\sigma$-compact subset of $A_{1}$, then $A_{2} \in \mathscr{F}$;

(R2) The union of each countable subcollection of $\mathscr{F}$ is also in $\mathscr{F}$;

(R3) If $A \in \mathscr{F}$ and $x \in G$, then $A+x \in \mathscr{F}$;

(R4) If $A \in \mathscr{F}$, then $A+A \epsilon \mathscr{F}$. A Raikov system $\mathscr{F}$ is said to be symmetric if the following additional condition is satisfied:

(R5) If $A \epsilon \mathscr{F}$, then $-A \epsilon \mathscr{F}$.

To each topology $\tau$ in $\mathscr{T}\left(G\left(\tau_{0}\right)\right)$ there corresponds the Raikov system $\mathscr{F}_{\tau}$ of all those subsets which are $\sigma$-compact with respect to $\tau$.

Let $\Phi$ be the homomorphism of $M(G(\tau))$ to $M\left(G\left(\tau_{0}\right)\right)$ which is induced by the canonical injective mapping of $G(\tau)$ to $G\left(\tau_{0}\right)$, then $\Phi(M(G(\tau)))$ $=M\left(\mathscr{F}_{\tau}\right)$ ([4]). Thus we may identify $M\left(\mathscr{F}_{\tau}\right)$ with $M(G(\tau))$. Each Raikov system $\mathscr{F}$ gives rise to an $L$-subalgebra $M(\mathscr{F})$ of all those measures which are concentrated on suitable sets in $\mathscr{F}$. This subalgebra is prime ([2], Section 33). 
In Section 2, by constructing a Raikov system of a special type, we shall show that there exists a non-trivial prime $L$-subalgebra which is different from $M(G(\tau))$ for all $\tau \in \mathscr{T}\left(G\left(\tau_{0}\right)\right)$. In this connection, we shall prove further that, if a symmetric Raikov system $\mathscr{F}_{1}$ with a single generator is contained properly in a Raikov system $\mathscr{F}_{2}$, then there is a $\mathrm{R}$ aik.ov system $\mathscr{F}$ which is contained properly in $\mathscr{F}_{2}$ and contains properly $\mathscr{F}_{1}$. In these constructions the notion of independent sets and that of semi-independent ones play a decisive role. The rest of the paper concerns construction of measures of special types in connection with independent sets. We prove the existence of a measure with independent powers. Finally, when the dual group admits an infinite independent set, then we can construct a measure which answers affirmatively to a problem raised by J. L. Taylor in [10].

1. Independent sets and prime $L$-subalgebras. Now we shall show that for a suitable symmetric Raikov system $\mathscr{F}, M(\mathscr{F})$ is a non-trivial prime $L$-subalgebra which is different from $M(G(\tau))$ for all $\tau \in \mathscr{T}\left(G\left(\tau_{0}\right)\right)$.

A Raikov system $\mathscr{F}$ such that $m_{\tau_{0}}(A)=0$ for every set $A \epsilon \mathscr{F}$, where $m_{\tau_{0}}$ is the Haar measure on $G\left(\tau_{0}\right)$, will be called a proper Raikov system. For a subset $E$ of $G$ and a positive integer $n$ we write that

$$
n E=\left\{x_{1}+\ldots+x_{n}: x_{1}, \ldots, x_{n} \in E\right\},
$$

and, in particular, we set $0 E=\{0\}$. If a Borel subset $E$ of $G\left(\tau_{0}\right)$ is locally negligible with respect to $m_{x}$, then we call $E$ locally $\tau$-negligible.

LEMMA 1.1. F.or any non-discrete topology $\tau \in \mathscr{T}\left(G\left(\tau_{0}\right)\right)$ and a positive number $n$, suppose that $E$ is a Borel subset such that $k E$ is a Borel subset of $G(\tau)$ for $k=1,2, \ldots, n$ and $n E$ is non-locally $\tau$-negligible. If $E^{\prime}$ is a subset of $E$ such that $E \backslash E^{\prime}$ is finite, then $n E^{\prime}$ has non-locally $\tau$-negligible Borel subsets.

Proof. Put $F=E \backslash E^{\prime}$, from $k E+(n-k) F \subset k E+(n-k) E=n E$, we have that $n E=\bigcup_{k=0}^{n}(k E+(n-k) F)$. Let $k_{0}$ be the smallest positive integer such that $k_{0} E+\left(n-k_{0}\right) F$ is non-locally $\tau$-negligible. Since $\left(n-k_{0}\right) F$ is finite, $k_{0} E$ is non-locally $\tau$-negligible. From

$$
k_{0} E=k_{0} E^{\prime} \cup \bigcup_{k=1}^{k_{0}}\left(\left(k_{0}-k\right) E^{\prime}+k Z^{\prime}\right),
$$

we have that

$$
k_{0} E \backslash \bigcup_{k=1}^{k_{0}}\left(\left(k_{0}-k\right) E+k F\right) \subset k_{0} E^{\prime}
$$

Since $\left(k_{0}-k\right) E+k F$ is locally $\tau$-negligible $\left(k=1,2, \ldots, k_{0}\right)$ and $k_{0} E^{\prime}$ $\subset n E^{\prime}, n E^{\prime}$ has a non-locally $\tau$-negligible Borel subset.
A subset $E$ of $G$ is said to be independent if $E$ has the following property: for every choice of distinct elements $x_{1}, \ldots, x_{k}$ of $E$ and integers $n_{1}, \ldots, n_{k}$, either

$$
n_{1} x_{1}+\ldots+n_{k} x_{k} \neq 0
$$

$$
n_{1} x_{1}=\ldots=n_{k} x_{k}=0 .
$$

LeMma 1.2. Let $P$ be an independent subset in $G$, and $Q=P \cup(-P)$. If $\tau \in \mathscr{T}\left(G\left(\tau_{0}\right)\right)$ is non-discrete and $k Q$ is a Borel subset in $G(\tau)$ for any positive integer $k$, then the group $G(P)$ generated by $P$ is locally $\tau$-negligible.

Proof. Since $G(P)=\bigcup_{k=1}^{\infty} k Q$, it is enough to show that $k Q$ is locally $\tau$-negligible for any positive integer $k$. Assume that for some fixed positive integer there is a $\tau$-compact subset $E$ of $k Q$ such that $E=-E$ and $m_{\tau}(E)>0$. If $\chi_{E}$ is the characteristic function of $E$ and $f=\chi_{E} * \chi_{E}$, then $f$ is continuous on $G(\tau)$ and $f(0)=m_{\tau}(E)>0$, hence $f(x)>0$ for all $x$ in some $\tau$-neighborhood $V$ of 0 which is contained in $2 E$ ([7], p. 108).

Let $U$ be a $\tau$-neighborhood of 0 such that $(2 k+1) U \subset V$. Since $G(\tau)$ is non-discrete, there is a non-empty finite subset $\left\{x_{1}^{(1)}, \ldots, x_{q_{1}}^{(1)}\right\}$ of $P$ such that $n_{1}^{(1)} x_{1}^{(1)}+\ldots+n_{q_{1}}^{(1)} x_{q_{1}}^{(1)}$ is a non-zero element of $U$ for some choice of non-zero integers $n_{1}^{(1)}, \ldots, n_{q_{1}}^{(1)}$.

Put $P_{1}=P \backslash\left\{x_{1}^{(1)}, \ldots, x_{q_{1}}^{(1)}\right\}$ and $Q_{1}=P_{1} \cup\left(-P_{1}\right)$. In view of Lemma 1.1, $k Q_{1}$ is non-locally $\tau$-negligible, thus $2 k Q_{1}$ contains a neighborhood of 0 in $G(\tau)$ ([7]). By induction, we can obtain distinct elements

$$
x_{1}^{(1)}, \ldots, x_{q_{1}}^{(1)}, \ldots, x_{1}^{(2 k+1)}, \ldots, x_{q_{2 k+1}}^{(2 k+1)}
$$

of $P$ and non-zero integers

such that

$$
n_{1}^{(1)}, \ldots, n_{q_{1}}^{(1)}, \ldots, n_{1}^{(2 k+1)}, \ldots, n_{q_{2 k+1}}^{(2 k+1)}
$$

$$
n_{1}^{(1)} x_{1}^{(1)}+\ldots+n_{q_{1}}^{(1)} x_{q_{1}}^{(1)}+\ldots+n_{1}^{(2 k+1)} x_{1}^{(2 k+1)}+\ldots+n_{q_{2 k+1}}^{(2 k+1)} x_{q_{2 k+1}}^{(2 k+1)}
$$

is an element of $(2 k+1) U$. On the other hand, from $(2 k+1) U \subset V \subset 2 k Q$, there are distinct elements $y_{1}, \ldots, y_{r} \in P \quad(1 \leqslant r \leqslant 2 k)$ and non-zero integers $m_{1}, \ldots, m_{r}$ such that

$$
\begin{aligned}
n_{1}^{(1)} x_{1}^{(1)}+\ldots+n_{q_{1}}^{(1)} x_{q_{1}}^{(1)}+\ldots+n_{1}^{(2 k+1)} x_{1}^{(2 k+1)}+\ldots+ & +n_{q_{2 k+1}}^{(2 k+1)} x_{q_{2 k+1}}^{(2 k+1)} \\
& =m_{1} y_{1}+\ldots+m_{r} y_{r} .
\end{aligned}
$$

This contradicts the independence of $P$.

THEOREM 1.3. Let $\mathscr{F}$ be a symmetric Raikov system generated by a compact independent set $P$. Then $M(\mathscr{F})$ is a symmetric prime $L$-subalgebra such that $M(\mathscr{F}) \perp L^{1}(G(\tau))$ for any non-discrete topology $\tau \in \mathscr{T}\left(G\left(\tau_{0}\right)\right)$, therefore 
$M(\mathscr{F})$ is a non-trivial prime L-subalgebra which is different from $M(G(\tau))$ for all $\tau \in \mathscr{T}\left(G\left(\tau_{0}\right)\right)$.

Proof. Let $H$ be the $\sigma$-compact group generated by $P$. Put $Q=P \cup$ $\cup(-P)$, then $k Q$ is $\tau_{0}$-compact, so that $k Q$ is $\tau$-closed for any $\tau \in \mathscr{T}\left(G\left(\tau_{0}\right)\right)$. Thus from Lemma 1.2, $H$ is locally $\tau$-negligible for any non-discrete topology $\tau \in \mathscr{T}\left(G\left(\tau_{0}\right)\right)$. Since any $A \epsilon \mathscr{F}$ is contained in the union of some countable cosets of $H, A$ is locally $\tau$-negligible, therefore $M(\mathscr{F}) \perp L^{1}(G(\tau))$ for any non-discrete topology $\tau \in \mathscr{T}\left(G\left(\tau_{0}\right)\right)$.

Our next purpose is to show the following theorem.

THEOREM 1.4. If $\mathscr{F}_{1}$ is a symmetric Raikov system generated by a $\sigma$-compact group $H$, and if $\mathscr{F}_{2}$ is a strictly larger symmetric Raikov system, then there exists a symmetric Raikov system $\mathscr{F}$ such that

(i) $M\left(\mathscr{F}_{1}\right) \varsubsetneqq M(\mathscr{F}) \varsubsetneqq M\left(\mathscr{F}_{2}\right)$ and

(ii) $M(\mathscr{F}) \neq M(G(\tau))$ for all $\tau \in \mathscr{T}\left(G\left(\tau_{0}\right)\right)$.

Given a subset $E$ of $G$, we say that a subset $P$ of $G$ is semi-E-independent, if $P$ has the property that for every choice of distinct points $x_{1}, \ldots, x_{N+1} \in P$ and integers $n_{1}, \ldots, n_{N}$ the relation

$$
\sum_{r=1}^{N} n_{r} x_{r}+x_{N+1} \notin E
$$

holds. In particular, if $E=\{0\}$, then we call briefly $P$ semi-independent.

LEMMA 1.5 (cf. [7], p. 108). Let $H$ be a $\sigma$-compact subgroup of $G\left(\tau_{0}\right)$ and $P$ a compact semi-H-independent subset of $G\left(\tau_{0}\right)$. If $P$ has a cluster point with respect to a topology $\tau \in \mathscr{T}\left(G\left(\tau_{0}\right)\right)$, then the group $\boldsymbol{H}+G(P)$ is locally $\tau$-negligible.

Proof. If $Q=P \cup(-P)$, then $H+G(P)=\bigcup_{k=1}^{\infty}(H+k Q)$. Suppose that $H+n Q$ is non-locally $\tau$-negligible for some integer $n$. Then there exists a $\tau$-compact subset $A$ of $H+n Q$ such that $m_{\tau}(A)>0$ and $A=-A$. We define the mapping $\psi$ of $G(\tau)^{2 n+2}$, the direct sum of $2 n+2$ copies of $G(\tau)$, to $G(\tau)$ as follows:

$$
\psi\left(\left(x_{1}, x_{2}, \ldots, x_{2 n+2}\right)\right)=\sum_{k=1}^{2 n+2}(-1)^{k} x_{k} .
$$
then

Let $x$ be a $\tau$-cluster point of $P$. Put $x_{l k}=x$ for $k=1,2, \ldots, 2 n+2$;

$$
\psi\left(\left(x_{1}, x_{2}, \ldots, x_{2 n+2}\right)\right)=0 .
$$

Since $m_{\tau}(A)>0, A+A$ contains a neighborhood $V$ of 0 with respect to $\tau$ ([7], p. 108). Since $x$ is a $\tau$-cluster point and $\psi$ is continuous, there is a element $\left(y_{1}, y_{2}, \ldots, y_{2 n+2}\right) \epsilon P^{2 n+2}$ such that $y_{i} \neq y_{j}$ if $i \neq j$, and $y_{1}-y_{2}+$ $+\ldots+y_{2 n+1}-y_{2 n+2} \in V$. From $V \subset A+A \subset H+2 n Q$, we can choose $w_{1}, \ldots, w_{2 n} \in Q$ and $h \in H$ such that

$$
y_{1}-y_{2}+\ldots+y_{2 n+1}-y_{2 n+2}=h+w_{1}+\ldots+w_{2 n} .
$$

It follows that

$$
r_{1} z_{1}+\ldots+r_{m-1} z_{m-1}+z_{m}=h,
$$

where $r_{1}, \ldots, r_{m-1}$ are integers, $z_{1}, \ldots, z_{m}$ are distinct elements of $P$. This contradicts the semi- $H$-independence of $P$.

ExaMPLe. For some locally compact abelian group $G\left(\tau_{0}\right)$, there is an example of a semi-independent compact perfect set $P$ of $G\left(\tau_{0}\right)$ such that $G(P)$ is open with respect to some non-discrete locally compact group topology on $G$. Let $G\left(\tau_{0}\right)=T \oplus D_{2}$, where $T$ is the circle group and $D_{2}$ is the complete direct sum of countable many copies of the cyclic group of order 2 ([7], p. 254). Let $P_{0}$ be an independent Cantor set of $D_{2}([7], \mathrm{p}$. 103). Let $\varphi_{1}$ be a homeomorphic mapping of $P_{0}$ onto $D_{2}$. Put $D_{2}=\prod_{n=1}^{\infty}\{0,1\}_{n}$. We define the mapping $\varphi_{2}$ of $D_{2}$ to $T$ as follows:

$$
\varphi_{2}\left(\left(x_{1}, x_{2}, \ldots\right)\right)=\sum_{n=1}^{\infty} x_{n} / 2^{n}
$$

for $\left(x_{1}, x_{2}, \ldots\right) \in D_{2}$. If we put

$$
\varphi(x)=\left(x, \varphi_{2} \circ \varphi_{1}(x)\right) \quad\left(x \in P_{0}\right),
$$

then $\varphi$ is a continuous mapping of $P_{0}$ to $D_{2} \oplus T$. Since $\left(\varphi_{2} \circ \varphi_{1}\right)^{-1}(t)$ is a finite set for any $t \in T, \varphi\left(P_{0}\right)$ has no $\tau_{T^{-}}$cluster point, where $\tau_{T}$ is the weakest locally compact group topology on $D_{2} \oplus T$ such that $T$ is open. Olearly, $\varphi\left(P_{0}\right)$ is a compact semi-independent subset of $D_{2} \oplus T$. Now, we have $\left\{2 x: x \in \varphi\left(P_{0}\right)\right\}=T$, thus the group generated by $\varphi\left(P_{0}\right)$ is $\tau_{T^{-}}$open.

LEMMA 1.6. Let $K$ be a compact subgroup of $G\left(\tau_{0}\right)$ and let a be the canonical homomorphism of $G\left(\tau_{0}\right)$ to $G\left(\tau_{0}\right) / K$. If $\mathscr{F}$ is a Raitiov system of $G\left(\tau_{0}\right)$, then $\alpha(\mathscr{F})=\{\alpha(A): A \epsilon \mathscr{F}\}$ is a Raikov system of $G\left(\tau_{0}\right) / K$.

Proof. It is clear that $\alpha(\mathscr{F})$ satisfies (R2), (R3) and (R4) of Section 0. Given a set $A \epsilon \mathscr{F}$, for any $\sigma$-compact set $B \subset \alpha(A)$, there is a $\sigma$-compact subset $B^{\prime}$ of $A$ such that $a\left(B^{\prime}\right)=B([6])$, and so $B \in \alpha(\mathscr{F})$.

LEMMA 1.7. Let $\mathscr{F}$ be a Raikov system generated by a $\sigma$-compact group $H$, and $A$ a compact perfect set such that $(H-x) \cap A$ is of the first category in $A$ for' each $x \in G\left(\tau_{0}\right)$. Then there is a compact group $K$ such that $G\left(\tau_{0}\right) / K$ is metrizable and $\alpha(A) \notin \alpha(\mathscr{F})$, where $\alpha$ is the canonical homomorphism of $G\left(\tau_{0}\right)$ to $G\left(\tau_{0}\right) / K$.

Proof. Suppose that $H=H_{1} \cup H_{2} \cup \ldots$, where each $H_{n}$ is compact and symmetric. We may assume $H_{1} \subset H_{2} \ldots$ We can choose compact 
$\tau_{0}$-neighborhoods $V_{n}$ and finite subsets $\left\{x_{1}^{(n)}, \ldots, x_{2}^{(n)}\right\}$ of $A(n=1,2, \ldots)$ such that

(i) $V_{n}=-V_{n}$,

(ii) $V_{n+1}+V_{n+1} \subset V_{n}$,

(iii) $x_{i}^{(n+1)}+V_{n+1} \subset x_{j}^{(n)}+V_{n}$ with $i \leqslant 2 j \leqslant i+1$,

(iv) $\left(\left(x_{i}^{(n)}+V_{n}\right)-\left(x_{j}^{(n)}+V_{n}\right)\right) \cap H_{n}=\varnothing$ if $i \neq j$ (cf. [8], [12]).

Put $\Lambda=\left\{\lambda=\left(\lambda_{1}, \lambda_{2}, \ldots\right): \lambda_{i}=0\right.$ or $\left.1(i=1,2, \ldots),\right\}$. For $\lambda \in A$ choose a set $\left\{x_{\lambda(1)}^{(1)}, x_{\lambda(2)}^{(2)}, \ldots\right\}$ with $\lambda(1)=2-\lambda_{1}$ and $\lambda(k)=2 \lambda(k-1)-\lambda_{k}$ $(k=2,3, \ldots)$. For $\lambda, \lambda^{\prime} \in \Lambda$, if $\lambda_{k} \neq \lambda_{k}^{\prime}$, then $\lambda(m) \neq \lambda^{\prime}(m)$ for every $m \geqslant k$. Let $K=\bigcap_{n=1}^{\infty} \nabla_{n}$, then, from (i) and (ii), $K$ is a compact group such that $G\left(\tau_{0}\right) / K$ is metrizable. Let $x_{\lambda}$ be a cluster point of $\left\{x_{\lambda(1)}^{(1)}, x_{\lambda(2)}^{(2)}, \ldots\right\}$, then, from (iii), $x_{\lambda} \epsilon \bigcap_{n=1}^{\infty}\left(\infty_{\lambda(n)}^{(n)}+V_{n}\right)$. Suppose $\lambda, \lambda^{\prime} \in \Lambda$ and $\lambda \neq \lambda^{\prime}$, then for some integer $k$ it follows that $\lambda(n) \neq \lambda^{\prime}(n)$ if $n \geqslant k$, so that

$$
\begin{aligned}
\left(\left(x_{\lambda}+K\right)-\left(x_{\lambda}^{\prime}+K\right)\right) & \cap H_{n} \\
& \subset\left(\left(x_{\lambda(n)}^{(n)}+V_{n+1}+K\right)-\left(x_{\lambda^{\prime}(n)}^{(n)}+V_{n+1}+K\right)\right) \cap H_{n} \\
& \subset\left(\left(x_{\lambda(n)}^{(n)}+V_{n+1}+V_{n+1}\right)-\left(x_{\lambda^{\prime}(n)}^{(n)}+V_{n+1}+V_{n+1}\right)\right) \cap H_{n} \\
& \subset\left(\left(x_{\lambda(n)}^{(n)}+V_{n}\right)-\left(x_{\lambda^{\prime}(n)}^{(n)}+V_{n}\right)\right) \cap H_{n}=\emptyset_{1}
\end{aligned}
$$

for every $n \geqslant k$. Thus $\lambda \neq \lambda^{\prime}$ implies

$$
\left(\left(x_{\lambda}+K\right)-\left(x_{\lambda^{\prime}}+K\right)\right) \cap H=\varnothing,
$$

that is,

Since $\Lambda$ is uncountable,

$$
x_{\lambda}-x_{\lambda^{\prime}} \notin H+K
$$

$$
a\left(\left\{x_{\lambda}\right\}_{\lambda \in \Lambda}\right) \notin \bigcup_{n=1}^{\infty}\left(\alpha(H)+y_{n}\right)
$$

for every countable set $\left\{y_{n}\right\}_{n=1}^{\infty}$ of $G\left(\tau_{0}\right) / K$. Thus $\alpha(A) \notin \alpha(\mathscr{F})$.

Proof of Theorem 1.4. Since $\mathscr{F}_{2}$ contains properly $\mathscr{F}_{1}$, there is a compact perfect set $A$ in $\mathscr{F}_{2}$ such that $(H-x) \cap A$ is of the first category in $A$ for each $x \in G$. (cf. [12]). From Lemma 1.7, there exists a compact group $K$ such that $G\left(\tau_{0}\right) / K$ is a metrizable group and $\alpha(A) \& \alpha\left(\mathscr{F}_{1}\right)$, where $\alpha$ is the canonical homomorphism of $G\left(\tau_{0}\right)$ to $G\left(\tau_{0}\right) / K$. From Lemma 1.6, $\alpha\left(\mathscr{F}_{2}\right)$ is a Raikov system which contains properly a Raikov system $\alpha\left(\mathscr{F}_{1}\right)$ generated by $\sigma$-compact group $\alpha(H)$. Since $G\left(\tau_{0}\right) / K$ is metrizable, there is a compact perfect semi- $\alpha(H)$-independent set $P \in a\left(\mathscr{F}_{2}\right) \backslash \alpha\left(\mathscr{F}_{1}\right)$. Let $P_{0}$ be a compact perfect subset such that $P \backslash P_{0}$ contains perfect subset. If $\mathscr{F}^{\prime}$ is a symmetric Raikov system which is generated by $\alpha(\boldsymbol{H})$ and $P_{0}$, then we have $\alpha\left(\mathscr{F}_{1}\right) \varsubsetneqq \mathscr{F}^{\prime} \varsubsetneqq \alpha\left(\mathscr{F}_{2}\right)$, because $\left(P \backslash P_{0}\right) \cap\left(G\left(P_{0}\right)+H+z\right)$ is a set consisting of at most one point for each $z \in G$. There is a compact set $P_{1} \in \mathscr{F}_{2}$ such that $\alpha\left(P_{1}\right)=P_{0}([6])$. Let $\mathscr{F}$ be the symmetric Raikov system generated by $P_{1}$ and $H$, then $\alpha(\mathscr{F})=\mathscr{F}^{\prime}$ and $\mathscr{F}_{1} \neq \mathscr{F}_{F} \mathscr{F}_{2}$.

The rest of the proof is to show that $M(\mathscr{F}) \neq M(G(\tau))$ for any $\tau$ $\epsilon \mathscr{T}\left(G\left(\tau_{0}\right)\right)$. It is enough to show that $M\left(\mathscr{F}^{\prime}\right) \neq M((G / K)(\tau))$ for any $\tau \in \mathscr{T}\left(G\left(\tau_{0}\right) / K\right)$ ([7], p. 54). If $P_{0}$ is a $\tau$-discrete set, then $M_{c}\left(P_{0}\right) \cap$ $\cap M((G / K)(\tau))=\{0\}$, where $M_{c}\left(P_{0}\right)$ is the subspace of $M \cdot\left(G\left(\tau_{0}\right) / K\right)$ consisting of all continuous measures whose supports are contained in $P_{0}$, so that in this case we have $M\left(\mathscr{F}^{\prime}\right) \neq M((G / K)(\tau))$. If $P_{0}$ has a $\tau$-cluster point, by Lemma 1.5, we obtain that $\alpha(H)+G\left(P_{0}\right)$ is locally $\tau$-negligible. Thus, it follows that $M\left(\mathscr{F}^{\prime}\right) \neq M((G / K)(\tau))$.

2. Independent power measures with respect to $M(\mathscr{F})$. If $f$ is a polynomial in elements of $M\left(G\left(\tau_{0}\right)\right)$, with coefficients $a_{r_{1} r_{2}} \ldots \in M\left(G\left(\tau_{0}\right)\right)$, we write $|f|$ for the polynomial with scalar coefficients $\left\|a_{r_{1}}, \ldots\right\|$. Let $M(\mathscr{F})$ be a given Raikor system. A set $X$ of non-zero measures $\left\{\mu_{i}\right\}$ has independent powers with respect to $M(\mathscr{F})$ if for each polynomial $f$ with coefficients in $M(\mathscr{F})$, we have

$$
\left\|f\left(\mu_{1}, \ldots, \mu_{n}\right)\right\|=|f|\left(\left\|\mu_{1}\right\|, \ldots,\left\|\mu_{n}\right\|\right)
$$

for all $\mu_{1}, \ldots, \mu_{n} \in X$ (cf. [11])

Throughout this section we shall assume that $G\left(\tau_{0}\right)$ is metrizable.

LEMTMA 2.1 ([8], [12]). Let $\mathscr{F}$ be a proper symmetric Raikov system which is generated by a $\sigma$-compact group $H$. Let $\left\{P_{i}\right\}_{n=1}^{\infty}$ be a disjoint collection of subsets of $G\left(\tau_{0}\right)$, with $P=\bigcup_{n=1}^{\infty} P_{i}$ semi-H-independent, and for each $i$ let $\mu_{i}$ be a continuous measure concentrated on $Q_{i}=P_{i} \cup\left(-P_{i}\right)$. If $a, b \epsilon$ $\epsilon M(\mathscr{F})$ and $\left(r_{1}, \ldots, r_{N}\right) \neq\left(s_{1}, \ldots, s_{N}\right)$, where $r_{1}, \ldots, r_{N}, s_{1}, \ldots, s_{N}$ are non-negative integers, then

$$
a * \mu_{1}^{r_{1} 1 * \ldots * \mu_{N}^{r_{N}} \perp b * \mu_{1}^{s_{1}} * \ldots * \mu_{N}^{s_{N}} .}
$$

Given a subset $E$ of $G$, we say (as in [12]) that a subset $X$ of $G$ is $(E, 2)$-independent if the relation

$$
n_{1} x_{1}+\ldots+n_{r} x_{r} \in E
$$

where $n_{1}, \ldots, n_{r}$ are integers satisfying

$$
\left|n_{i}\right| \leqslant 2 \quad(1 \leqslant i \leqslant r)
$$

and $x_{1}, \ldots, x_{r}$ are distinct elements of $X$, is possible only if $n_{1} x_{1}=\ldots$ $\ldots=n_{r} x_{r}=0$. For a given subset $E$ of $G$, we put

$$
2 \times E=\{2 x: x \in E\} \text {. }
$$

The proof of the following proposition is essentially the same as that of Proposition 2 in [12]. 
PRoposinton 2.2. Let $\mathscr{F}$ be a proper symmetric Raikov system generated by a $\sigma$-compact group $H$. Let $\mu_{i}(i=1, \ldots, r)$ be mutually singular continuous measures which are concentrated on $P \cup(-P)$. If $P$ is $(H, 2)$-independent, then the set of measures $\left\{\mu_{i}\right\}_{i=1}^{r}$ has independent powers with respect to $M(\mathscr{F})$

THEOREM 2.3. Let $\mathscr{F}_{1}$ be a Raikov system generated by a $\sigma$-compact group $H$, and $\mathscr{F}_{2}$ a strictly larger symmetric Raikov system. Then $2 \times H=H$ implies that there is a compact perfect $(H, 2)$-independent set $P$ in $\mathscr{F}_{2}$, so that the set of continuous measures $\left\{\mu_{i}\right\}_{i=1}^{n}$ in $M\left(\mathscr{F}_{2}\right)$, which are concentrated on $P \cup(-P)$ and are mutually singular, has independent powers with respect to $M\left(\mathscr{F}_{1}\right)$.

Proof. Let $\mathscr{P}$ be the family consisting of all compact perfect semi-Hindependent sets which belong to $\mathscr{F}_{2}$, then $\mathscr{P}$ is non-empty (cf. [8], [12]). If $2 \times P^{\prime} \notin H$ for each $P^{\prime} \in \mathscr{P}$, then it is easy to show the existence of compact perfect $(H, 2)$-independent sets (cf. [12]). We consider the case that $2 \times P_{0} \subset H$ for some $P_{0} \in \mathscr{P}$. Let $G_{2}=\left\{x \in G\left(\tau_{0}\right): 2 x=0\right\}$. Since $2 \times P_{0}$ $\subset 2 \times H=H$, for each $p \in P_{0}$ there exists $h \in H$ such that $p-h \in G_{2}$. Then we have that $\left(P_{0}-H\right) \cap G_{2} \in \mathscr{F}_{2} \backslash \mathscr{F}_{1}$. In fact, suppose $\left(P_{0}-H\right) \cap G_{2} \subset$ $\bigcup_{n=1}^{\infty}\left(H+x_{n}\right)$ for some countable set $\left\{x_{n}\right\}_{n=1}^{\infty}$. For any $p \in P_{0}$ there is an element $h$ of $H$ such that $p-h \in G_{2}$, so that $p-h \epsilon \bigcup^{\infty}\left(H+x_{n}\right)$. Since $H$ is a group, $p \in \bigcup_{n=1}^{\infty}\left(H+x_{n}\right)$, that is, $P_{0} \subset \bigcup_{n=1}^{\infty}\left(H+x_{n}\right)$. On the other hand, by Lemma $2.1, P_{0} \in \mathscr{F}_{2} \backslash \mathscr{F}_{1}$, this is a contradiction. Choose a compact perfect semi- $H$-independent subset $P$ of $\left(P_{0}-H\right) \cap G_{2}$ (cf. [12]). Since $2 \times G_{2}=\{0\}$, every semi- $H$-independent subset $P$ of $G_{2}$ is $(H, 2)$-independent. Thus $P$ is a compact perfect $(H, 2)$-independent subset in $\mathscr{F}_{2}$. By Prop osition 2.2 , if $\left\{\mu_{i}\right\}_{i=1}^{n}$ is a set of continuous measures which are concentrated on $P \cup(-P)$, then $\left\{\mu_{i}\right\}_{i=1}^{n}$ has independent powers with respect to $M\left(\mathscr{F}_{1}\right)$.

EXAMPLE. There is an example of a locally compact abelian group $G\left(\tau_{0}\right)$ such that the statement of Proposition 2.2 is not established, that is, there is a Raikov system $\mathscr{F}$ of $G\left(\tau_{0}\right)$ generated by a $\sigma$-compact group $H$ such that for any compact perfect semi- $H$-independent set $P$ every non-zero continuous Hermitian measure $\mu$ concentrated on $P \cup(-P)$ does not have independent powers with respect to $M(\mathscr{F})$.

Let $\left\{Z_{4}^{(n)}\right\}_{n=1}^{\infty}$ be the family of cyclic groups of order 4 and $Z_{2}^{(n)}$ the subgroup of $Z_{4}^{(n)}$ of order 2 . We shall define the groups as follows $G\left(\tau_{0}\right)$ $=\prod_{n=2}^{\infty} Z_{4}^{(n)}$ and $H=\prod_{n=2}^{\infty} Z_{2}^{(n)}$. Let $P$ be any compact perfect semi- $H$-independent set of $G\left(\tau_{0}\right)$. Then we can assume that $P \subset\left\{a_{1}^{(1)}\right\} \times \prod_{n=1}^{\infty} Z_{4}^{(n)}$, where $a_{1}^{(1)}$ is an element of $Z_{4}^{(1)}$ of order 4. Let $a_{2}^{(1)}$ is a non-zero element of $Z_{2}^{(1)}$.
If $m_{0}$ is the normalized Haar measure on $H_{1}=\left\{a_{0}^{(1)}\right\} \times \prod_{n=2}^{\infty} Z_{2}^{(n)}$, where $a_{0}^{(1)}$ is a unit element of $Z_{2}^{(1)}$, then it is clear that

$$
m_{0} \perp m_{0} * \delta_{a_{2}^{(1)}}
$$

where $\delta_{a_{2}}^{(1)}$ is the probability measure concentrated at the point $a_{2}^{(1)}$. Let $\mu$ be any continuous probability measure which is concentrated on $P$. We shall show that

$$
m_{0} * \mu \text { non } \perp m_{0} * \delta_{a_{2}^{(1)} * \mu^{*}} .
$$

If $E_{0}$ and $E_{1}$ are any Borel sets on which $m_{0} * \mu$ and $m_{0} * \delta_{a_{2}^{(1)}} * \mu^{*}$ are concentrated respectively, then we have

$$
m_{0} * \mu\left(E_{0}\right)=\int m_{0}\left(E_{0}-x\right) d \mu(x)=1 .
$$

Write $A_{0}=\left\{x \in P: m_{0}\left(E_{0}-x\right)=1\right\}$; it follows that

and

$$
\mu\left(A_{0}\right)=1
$$

$$
m_{0}\left(H_{1} \backslash\left(E_{0}-x\right)\right)=0 \quad \text { for each } x \in A_{0} .
$$

Similarly, we get

$$
\left.m_{0} * \delta_{a_{2}^{(1)}} \mid\left(H_{1}+a_{2}^{(1)}\right) \backslash\left(E_{1}+x\right)\right)=0 \quad \text { for each } x \in A_{1},
$$

where $A_{1}=\left\{x \in P: m_{0} * \delta_{a_{2}}^{(1)}\left(E_{1}+x\right)=1\right\}$. From $\mu\left(A_{0}\right)=\mu\left(A_{1}\right)=1$, it follows that $A_{0} \cap A_{1}$ is non-empty. Given $x_{0} \in A_{0} \cap A_{1}$, then we have

and so

$$
H_{1}+x_{0}=H_{1}+a_{2}^{(1)}-x_{0},
$$

Thus, we have

$$
m_{0} * \delta_{x_{0}}=m_{0} * \delta_{a_{2}^{(1)} * \delta_{-x_{0}}} .
$$

$$
m_{0} * \delta_{x_{0}}\left(\left(H_{1}+x_{0}\right) \backslash E_{0}\right)=m_{0} * \delta_{a_{2}^{(1)}} * \delta_{-x_{0}}\left(\left(H_{1}+a_{2}^{(1)}-x_{0}\right) \backslash E_{1}\right)=0 .
$$

Hence, it follows that $E_{0} \cap E_{1} \neq \varnothing$. This shows that $m_{0} * \mu$ non $\perp m_{0} * \delta_{a_{2}^{(1)}} * \mu *$. Thus, it follows that

$$
\left\|m_{0} * \mu-m_{0} * \delta_{a_{2}^{(1)}} * \mu^{*}\right\|<\left\|m_{0} * \mu\right\|+\| m_{0} * \delta_{a_{2}^{(1)} * \mu^{*}\|=2\| m_{0}\|\| \mu \| .}
$$

Define the polynomial $f$ in elements of $M\left(G\left(\tau_{0}\right)\right)$ as follows

$$
f(\nu)=\left(m_{0}-m_{0} * \delta_{\left.a_{2}^{(1)}\right) * \nu} \quad\left(\nu \in M\left(G\left(\tau_{0}\right)\right)\right) .\right.
$$


From $m_{0} \perp m_{0} * \delta_{a_{2}}^{(1)}$ and $\mu$ being non-negative,

$$
|f|\left(\left\|\mu+\mu^{*}\right\|\right)=4\left\|m_{0}\right\|\|\mu\| .
$$

On the other hand,

$$
\begin{aligned}
\left\|f\left(\mu+\mu^{*}\right)\right\| & =\left\|m_{0} * \mu-m_{0} * \delta_{a_{2}^{(1)} *} *\right\|+\left\|m_{0} * \mu^{*}-m_{0} * \delta_{a_{2}^{(1)}} * \mu\right\| \\
& <2\left\|m_{0}\right\|\|\mu\|+\left\|m_{0} * \mu^{*}\right\|+\left\|m_{0} * \delta_{a_{2}^{(1)}}^{(1)} \mu\right\|=4\left\|m_{0}\right\|\|\mu\| .
\end{aligned}
$$

Therefore, it follows that

$$
\left\|f\left(\mu+\mu^{*}\right)\right\|<|f|\left(\left\|\mu+\mu^{*}\right\|\right),
$$

that is, every non-zero continuous Hermitian measure concentrated on $P \cup(-P)$ does not have independent powers with respect to $M(\mathscr{F})$, where $\mathscr{F}$ is the Raikor system generated by $H=\prod_{n=1}^{\infty} Z_{2}^{(n)}$.

Next we shall show the following theorem.

THEOREM 2.4. Let $\mathscr{F}_{1}$ be a symmetric Raikov system generated by a com pact independent set $P_{1}$, and $F_{2}$ a strictly larger symmetric Raikov system. If there exists a compact set $A \in \mathscr{F}_{2} \backslash \mathscr{F}_{1}$ such that $2 \times A \subset P_{1}$, then there exists a compact perfect set $P$ in $\mathscr{F}_{2}$ such that any non-zero continuous measure $\mu$ concentrated on $P \cup(-P)$ has independent powers with respect to $M\left(\mathscr{F}_{1}\right)$.

We shall prove the next lemma to show this theorem.

LEMMA 2.5. For a $\sigma$-compact subgroup $H$ of $G\left(\tau_{0}\right)$, let $P$ be a compact semi-H-independent set such that $2 \times P \subset H$. Suppose that $a_{1}$ and $a_{2}$ are concentrated on $H-z$ and $H$, respectively; then $(H-z) \cap H=\emptyset$ implies that

$$
a_{1} * \mu^{n} \perp a_{2} * \mu^{n} \quad(n=1,2, \ldots)
$$

for any continuous measure $\mu$ which is concentrated on $P \cup(-P)$.

Proof. The measures $a_{1} * \mu^{n}$ and $a_{2} * \mu^{n}$ are concentrated on $H-z+$ $+n(P \cup(-P))$ and $H+n(P \cup(-P))$, respectively. Evidently, if the sets are not disjoint, we have

$$
h_{1}+x_{1}+\ldots+x_{n}-z=h_{2}+y_{1}+\ldots+y_{n}
$$

for some $h_{1}, h_{2} \in H$ and $x_{1}, \ldots, x_{n}, y_{1}, \ldots, y_{n} \in P \cup(-P)$.

Denote by $S$ the set of points $\left(x_{1}, \ldots, x_{n}\right) \in P \cup(-P) \times \ldots \times P \cup(-P)$ such that for some $h \in H$ we have

$$
h+x_{1}+\ldots+x_{n} \epsilon H+n(P \cup(-P))+z .
$$

Since $H$. is a group, $S$ is the set of points $\left(x_{1}, \ldots, x_{n}\right) \in P \cup(-P) \times \ldots$ $\ldots \times P \cup(-P)$ such that

$$
x_{1}+\ldots+x_{n} \epsilon H+n(P \cup(-P))+z .
$$

If we can show that $\mu \times \ldots \times \mu(S)=0$, then it will follows that $a_{1} \times \mu \times \ldots$ $\ldots \times \mu((H-z) \times S)=0$, which implies that $a_{1} * \mu^{n}(H+n(P \cup(-P)))=0$. Let $z=h^{\prime}+y_{1}^{\prime}+\ldots+y_{n}^{\prime}-x_{1}^{\prime}-\ldots-x_{n}^{\prime}$ with $h^{\prime} \in H$ and $y_{1}^{\prime}, \ldots, y_{n}^{\prime}, x_{1}^{\prime}, \ldots$ $\ldots, x_{n}^{\prime} \in P \cup(-P)$. If $\left(x_{1}, \ldots, x_{n}\right) \in S$, then

$$
z=h+x_{1}+\ldots+x_{n}-y_{1}-\ldots-y_{n}
$$

for some $h \in H$ and $y_{1}, \ldots, y_{n} \in P \cup(-P)$. Thus we have that

$$
x_{1}+\ldots+x_{n}-y_{1}-\ldots-y_{n}+x_{1}^{\prime}+\ldots+x_{n}^{\prime}-y_{1}^{\prime}-\ldots-y_{n}^{\prime} \epsilon H .
$$

Since the set $P$ is semi- $H$-independent and $2 \times P \subset H$ and $z \notin H$, the set $S$ is contained in a finite union of sets of the form

$$
\begin{aligned}
& A_{1}=\bigcup_{1 \leqslant i \neq j \leqslant n}\left\{\left(x_{1}, \ldots, x_{n}\right): x_{i}=x_{j} \text { or }-x_{j}\right\}, \\
& A_{2}=\bigcup_{1 \leqslant i, j \leqslant n}\left\{\left(x_{1}, \ldots, x_{n}\right): x_{i}=x_{j}^{\prime} \text { or }-x_{j}^{\prime}\right\}, \\
& A_{2}=\bigcup_{1 \leqslant i, j \leqslant n}\left\{\left(x_{1}, \ldots, x_{n}\right): x_{i}=y_{j}^{\prime} \text { or }-y_{j}^{\prime}\right\} .
\end{aligned}
$$

Since $\mu$ is continuous, these sets are of $(\mu \times \ldots \times \mu)$-measure zero. It follows that $a_{1} * \mu^{n}(H+n(P \cup(-P)))=0$, and so $a_{1} * \mu^{n}$ and $a_{2} * \mu^{n}$ are mutually singular.

For a given compact subset $Q$ of $G\left(\tau_{0}\right)$ and non-negative integer $n$, we call a measure $\mu$ of order $n Q$ provided that $\mu$ is concentrated on $n Q$ and $\mu(k Q)=0$ if $0 \leqslant k \leqslant n-1$.

Proof of Theorem 2.4. Let $Q_{1}=P_{1} \cup\left(-P_{1}\right)$ and $H=\bigcup_{n=1}^{\infty} n Q_{1}$. We can choose a compact perfect semi- $H$-independent subset $P$ of $A$. Let $\mu$ be a non-zero continuous measure concentrated on $P \cup(-P)$. Take mutually singular measures $\omega_{1}$ and $\omega_{2}$ which are concentrated on $H$. We have to show that

$$
\omega_{1} * \mu^{n} \perp \omega_{2} * \mu^{n} \quad(n=1,2, \ldots) .
$$

Without loss of generality we may assume that $\omega_{i}$ have order $r_{i} Q_{1}(i=1$, 2 ), with $r_{1} \geqslant r_{2}$. Let $A^{(1)}$ and $A^{(2)}$ be mutually disjoint Borel sets on which $\omega_{1}$ and $\omega_{2}$ are concentrated, respectively. For any $x \in A^{(1)}$, denote by $\mathcal{S}^{(x)}$ the set of points $\left(q_{1}, \ldots, q_{n}\right) \in Q \times \ldots \times Q$ such that for some $y \in A^{(2)}$ and $q_{1}^{\prime}, \ldots, q_{n}^{\prime} \in Q$, we have

$$
x+q_{1}+\ldots+q_{n}=y+q_{1}^{\prime}+\ldots+q_{n}^{\prime} .
$$

We may assume that $q_{1}, \ldots, q_{n}$ are all different, and so are $q_{1}^{\prime}, \ldots, q_{n}^{\prime}$, since $\mu$ is continuous. We can write the elements $x$ and $y$ as follows:

and

$$
x=m_{1}^{(x)} p_{1}^{(x)}+\ldots+m_{k(x)}^{(x)} p_{k(x)}^{(x)}
$$$$
y=m_{1}^{(y)} p_{1}^{(y)}+\ldots+m_{k(y)}^{(y)} p_{k(y)}^{(y)},
$$ 
where

$$
\begin{gathered}
p_{1}^{(x)}, \ldots, p_{k(x)}^{(x)}, p_{1}^{(y)}, \ldots, p_{k(y)}^{(y)} \in P_{1}, \\
\left|m_{1}^{(x)}\right|+\ldots+\left|m_{k(x)}^{(x)}\right|=r_{1} \text { and }\left|m_{1}^{(y)}\right|+\ldots+\left|m_{k(y)}^{(y)}\right|=r_{2} .
\end{gathered}
$$

Then we get

$$
\begin{aligned}
q_{\mathbf{1}}+\ldots+ & q_{n}-q_{1}^{\prime}-\ldots-q_{n}^{\prime} \\
& =m_{1}^{(y)} p_{1}^{(y)}+\ldots+m_{k(y)}^{(y)} p_{k(y)}^{(y)}-m_{1}^{(x)} p_{1}^{(x)}-\ldots-m_{k(x)}^{(x)} p_{k(x)}^{(x)} \neq 0 .
\end{aligned}
$$

From $P$ being semi- $H$-independent, it follows that

$$
2 q_{1}^{\prime \prime}+\ldots+2 q_{r}^{\prime \prime}-m_{1}^{(y)} p_{1}^{(y)}-\ldots-m_{k(y)}^{(y)} p_{k(y)}^{(y)}+
$$

$$
+m_{1}^{(x)} p_{1}^{(x)}+\ldots+m_{k(x)}^{(x)} p_{k(x)}^{(x)}=0,
$$

with $\left\{q_{1}^{\prime \prime}, \ldots, q_{r}^{\prime \prime}\right\} \subset\left\{q_{1}, \ldots, q_{n}\right\}$. Then, since $2 \times P \subset P_{1}, r_{1} \geqslant r_{2}$ and $P_{1}$ is independent, $S^{(x)}$ is contained in a finite union of sets of the form

$$
S_{(j, r)}^{(x)}=\left\{\left(q_{1}, \ldots, q_{n}\right) \in S^{(x)}: 2 q_{j}= \pm p_{r}^{(x)}\right\} \quad(j=1, \ldots, n, r=1, \ldots, k(x)) .
$$

If $\mu\left(\left\{q \in Q: 2 q= \pm p_{r}^{(x)}\right\}\right) \neq 0$, then there is a compact perfect $(H, 2)$ independent subset in $\left\{q \in Q: 2 q=p_{r}^{(x)}\right\}-q_{0}$, with $2 q_{0}= \pm p_{r}^{(x)}$. Thus, in this case, the statement of this proposition is established. If $\mu\left\{\left(q \in Q^{(1)}\right.\right.$. $\left.\left.2 q=p_{r}^{(x)}\right)\right\}=0$ for all $x \in A^{(1)}$, then

$$
\begin{aligned}
& \int \chi_{\left(\mathcal{A}^{(1)}+n Q\right)}^{(x) \cap\left(\mathcal{A}^{(2)}+n Q\right)} d \omega_{1} * \mu^{n}(x) \\
& \quad=\int \chi_{\mathcal{A}^{(1)}(x)}\left\{\int \ldots \int \chi_{\left.S^{(}\right)}\left(q_{1}, \ldots, q_{n}\right) d \mu\left(q_{1}\right) \ldots d \mu\left(q_{n}\right)\right\} d \omega_{1}(x)=0,
\end{aligned}
$$

and so $\omega_{1} * \mu^{n} \perp \omega_{2} * \mu^{n}$. Thus, on the basis of Lemma 2.1 and Lemma 2.3 , the rest of the proof is quite similar to that of the analogous part of Proposition 2 in [12].

3. Independent sets and certain measures. In [9] J. L. Taylor showed that there exists a compact commutative topological semigroup $S$ with identity and an order preserving isometric isomorphism $\theta$ of $M\left(G\left(\tau_{0}\right)\right.$ into $M(S)$, where $M(S)$ is the Banach algebra consisting of all bounded regular Borel measures on $S$, such that

(1) the image of $M\left(G\left(\tau_{0}\right)\right)$ in $M(S)$ is wealk*-dense;

(2) each non-zero multiplicative linear functional $h$ on $M\left(G\left(\tau_{0}\right)\right)$ has the form $h(\mu)=\int_{S} f d \theta \mu$ for some non-zero continuous semicharacter. $f$ on $S$;

(3) there are enough non-zero continuous semicharacters on $S$ to separate points; and

(4) if $\mu \epsilon M(G), \nu \in M(S)$ and $\nu \ll \theta \mu$, then there is a measure $\omega \in M(G)$ such that $\omega \ll \mu$ and $\theta \omega=\nu$.
We call $S$ the structure semigroup of $M\left(G\left(\tau_{0}\right)\right)$. The space of all nonzero semicharacters on $S$ is denoted by $\hat{S .}$ We may consider $\hat{S}$ to be the maximal ideal space of $M\left(G\left(\tau_{0}\right)\right)$.

Given an idempotent $p$ of $S$, let $K_{p}$ denote the maximal group of $S$ with $p$ as unit, and $N_{p}$ the set of those measures $\mu$ in $M\left(G\left(\tau_{0}\right)\right)$ for which $\theta \mu$ are concentrated on $K_{p}$. In [10] Taylor showed that if $N_{p}$ is non-trivial then there is a topology $\tau \in \mathscr{T}\left(G\left(\tau_{0}\right)\right)$ such that $N_{p}$ coincides with the radical $L^{1 / 2}(G(\tau))$ of $L^{1}(G(\tau))$ in $M(G(\tau))$, the intersection of all maximal ideals containing the ideal $L^{1}(G(\tau))$. Let $K$ stand for the union of all $K_{p}$, where $p$ runs over the set of idempotents of $S$. We shall denote by $M_{K}\left(G\left(\tau_{0}\right)\right)$ the set of all those measures $\mu$ in $M\left(G\left(\tau_{0}\right)\right)$ for which $\theta \mu$ are concentrated on $K$ but vanish on $K_{p}$ for every idempotent $p$.

The purpose of this section is to show that under suitable restriction $M_{K}\left(G\left(\tau_{0}\right)\right)$ is not trivial. This will give an affirmative answer to the problem raised by Taylor in [10]. It should be remarked that K. Izuchi ([5]) also proved independently the non-triviality of $M_{K}\left(G\left(\tau_{0}\right)\right)$ for the case of the Bohr compactification of the real line group.

Let us introduce several notations. Let

$\Lambda_{n}=\left\{\left(\alpha_{0}, \alpha_{1}, \alpha_{2}, \ldots\right): \alpha_{0}=1, \alpha_{j}=1\right.$ or 2 for $1 \leqslant j \leqslant n$ and $\alpha_{j}=0$ for $n+1 \leqslant j\}$

and $\Lambda=\bigcup_{n=0}^{\infty} \Lambda_{n}$. For $\alpha \in \Lambda$ we write $|\alpha|=n$ if $a$ belongs to $\Lambda_{n}$. Let $\alpha$ $=\left(\alpha_{0}, \alpha_{1}, \ldots\right)$ and $\beta=\left(\beta_{0}, \beta_{1}, \ldots\right)$ be elements of $\Lambda$. If $\alpha_{j}=\beta_{j}(0 \leqslant j \leqslant n)$ and $a_{n+1} \neq \beta_{n+1}$, then we denote by $\alpha \wedge \beta$ the element $\left(\alpha_{0}, a_{1}, \ldots, \alpha_{n}\right.$, $0,0, \ldots)$. The notation $\alpha \geqslant \beta$ will mean the relation $\alpha \wedge \beta=\beta$. If $\alpha \neq \beta$, then $\alpha^{\prime} \wedge \beta^{\prime}=\alpha \wedge \beta$ whenever $\alpha \leqslant \alpha^{\prime}$ and $\beta \leqslant \beta^{\prime}$.

Let $G\left(\tau_{0}\right)$ be the complete direct sum of a family $\left\{H_{\alpha}\right\}_{a \in \Lambda}$ of infinite compact groups, in particular, $H_{a}$ is uncountable. Each $x \in G\left(\tau_{0}\right)$ may be thought of as a string $x=\left(\ldots, x_{\alpha}, \ldots\right)$, the group operating being componentwise addition. For each $\alpha, \in \Lambda$ let

$$
G_{a_{0}}=\left\{\left(\ldots, x_{\beta}, \ldots\right) \in G\left(\tau_{0}\right): x_{\beta}=0 \text { if } \beta \leqslant \alpha_{0}\right\} .
$$

Let $m_{\alpha}$ be the normalized Haar measure on $G_{\alpha}$. We define the measure $\mu_{n}(n=1,2, \ldots)$ as follows,

$$
\mu_{n}=\frac{1}{2^{n}} \sum_{a \in \Lambda_{n}} m_{\alpha} .
$$

LEMMA 3.1. The countable set $\left\{\mu_{n}\right\}_{n=1}^{\infty}$ has the unique weak-* cluster point.

Proof. Since $G\left(\tau_{0}\right)$ is compact, by the uniqueness of Fourier-Stieltjes transform, if $\left\{\hat{\mu}_{n}(\gamma)\right\}_{n=1}^{\infty}$, where $\hat{\mu}_{n}$ is the Fourier-Stieltjes transform of 
$\mu_{n}$, is convergent for each continuous character $\gamma$ of $G\left(\tau_{0}\right)$, then $\left\{\mu_{n}\right\}_{n=0}^{\infty}$ has the unique weak-* cluster point. If $\alpha, \beta \in \Lambda$ and $\beta \leqslant \alpha$, then $G_{\alpha} \subset G_{\beta}$. Since $\hat{m}_{\alpha}(\gamma)=1$ if $\gamma=1$ on $G_{\alpha}$ and $\hat{m}_{a}(\gamma)=0$ if $\gamma \neq 1$ on $G_{\alpha}([7]$, p. 10), $\beta \leqslant \alpha$ implies

$$
\hat{m}_{\beta}(\gamma) \leqslant \hat{m}_{\alpha}(\gamma)
$$

Thus, if $1 \leqslant n \leqslant m$, then

$$
\begin{aligned}
\hat{\mu}_{m}(\gamma)=\frac{1}{2^{m}} \sum_{a \in \Lambda_{m}} \hat{m}_{\alpha}(\gamma) & =\frac{1}{2^{m}} \sum_{\beta \in \Lambda_{n}} \sum_{\beta \leqslant \alpha \in \Lambda_{m}} \hat{m}_{\alpha}(\gamma) \\
& \geqslant \frac{1}{2^{m}} \sum_{\beta \in \Lambda_{n}} 2^{m-n} \hat{m}_{\beta}(\gamma)=\frac{1}{2^{n}} \sum_{\beta \in \Lambda_{n}} \hat{m}_{\beta}(\gamma)=\hat{\mu}_{n}(\gamma),
\end{aligned}
$$

so that $\left\{\hat{\mu}_{n}(\gamma)\right\}_{n=1}^{\infty}$ is a non-decreasing sequence. Thus $\left\{\hat{\mu}_{n}(\gamma)\right\}_{n=1}^{\infty}$ is convergent for each continuous character $\gamma$ of $G\left(\tau_{0}\right)$. This completes the proof.

Let $\mu$ be the weak-* limit of $\left\{\mu_{n}\right\}_{n=1}^{\infty}$, then clearly $\mu$ is a probability measure. Given $\alpha \in \Lambda$ and an integer $n \geqslant|\alpha|$, we put

$$
\mu_{n}^{a}=\frac{1}{2^{n}} \sum_{\beta \in \Lambda_{n}^{\alpha}} m_{\beta},
$$

where $\Lambda_{n}^{a}=\left\{\beta \in \Lambda_{n}: \alpha \leqslant \beta\right\}$. Then $\left\{\mu_{n}^{\alpha}\right\}_{n=|a|}^{\infty}$ has the unique weak-* cluster point $\mu^{\alpha}$ with the norm $\frac{1}{2^{|\alpha|}}$ whose support is contained in $G_{a}$. Furthermore, we obtain

$$
\mu=\sum_{\alpha \in \Lambda_{n}} \mu^{\alpha} \quad(n=1,2, \ldots) .
$$

LEMMA 3.2. If $\alpha \neq \beta$ and $|\alpha|=|\beta|$, then

$$
\mu^{\alpha} * \mu^{\beta}=\frac{1}{2^{2|\alpha|}} m_{\alpha \wedge \beta}
$$

Proof. Since $\alpha^{\prime} \geqslant \alpha$ and $\beta^{\prime} \geqslant \beta$ implies

it follows that

$$
\alpha^{\prime} \wedge \beta^{\prime}=\alpha \wedge \cdot \beta \quad \text { and } \quad m_{\alpha^{\prime}} * m_{\beta^{\prime}}=m_{\alpha^{\prime} \wedge \beta^{\prime}}=m_{\alpha \wedge \beta},
$$

$$
\begin{aligned}
\mu_{n}^{\alpha} * \mu_{n}^{\beta} & =\left\{\frac{1}{2^{n}} \sum_{a^{\prime} \in \Lambda_{n}^{\alpha}} m_{\alpha^{\prime}}\right\} *\left\{\frac{1}{2^{n}} \sum_{\beta^{\prime} \in \Lambda_{n}^{\alpha}} m_{\beta^{\prime}}\right\} \\
& =\frac{1}{2^{2 n}} \sum_{\alpha^{\prime} \in \Lambda_{n}^{\alpha}} \sum_{\beta^{\prime} \in \Lambda_{n}^{\beta}} m_{\alpha^{\prime}} * m_{\beta^{\prime}}=\frac{1}{2^{2|a|}} m_{\alpha \wedge \beta} .
\end{aligned}
$$

LEMMA 3.3. The measure $\theta \mu$ is concentrated on $K$.

Proof. It is enough to prove

$$
\theta \mu\left(A_{f}\right)=0 \quad \text { for each } f \in \hat{S},
$$

where $A_{f}$ is the set of those points $s$ of $S$ for which $0<|f(s)|<1$ ([10]). If $a, \beta \epsilon \Lambda_{n}$ and $a \neq \beta$, then the inequality, which is a consequence of multiplicativity of $f$,

$$
\chi_{A_{f}}(x) \chi_{A_{f}}(y) \leqslant \chi_{A_{f}}(x y)
$$

holds and Lemma 3.2 implies

$$
\begin{aligned}
0 & \leqslant \theta \mu^{\alpha}\left(A_{f}\right) \theta \mu^{\beta}\left(A_{f}\right) \\
& =\iint \chi_{A_{f}}(x) \chi_{A_{f}}(y) d \theta \mu^{\alpha}(x) d \theta \mu^{\beta}(y) \\
& \leqslant \iint \chi_{A_{f}}(x y) d \theta \mu^{\alpha}(x) d \theta \mu^{\beta}(y) \\
& =\theta \mu^{\alpha} * \theta \mu^{\beta}\left(A_{f}\right)=\theta\left(\mu^{\alpha} * \mu^{\beta}\right)\left(A_{f}\right)=\frac{1}{2^{2 n}} \theta m_{a \wedge \beta}\left(A_{f}\right) .
\end{aligned}
$$

Here the multiplicativity of the map $\theta$ is important. Since the measure $m_{a \wedge \beta}$ is concentrated on $K([10])$, it follows that

$$
\theta \mu^{a}\left(A_{f}\right) \theta \mu^{\beta}\left(A_{f}\right)=0 .
$$

hence $\theta \mu^{\alpha}\left(A_{f}\right)=0$ or $\theta \mu^{\beta}\left(A_{f}\right)=0$. Then, since $\mu=\sum_{a \in A_{n}} \mu^{\alpha}$, for each $n$, there is $\alpha \in \Lambda_{n}$ such that

$$
\theta \mu\left(A_{f}\right)=\theta \mu^{\alpha}\left(A_{f}\right) \leqslant \frac{1}{2^{n}}
$$

This leads to $\theta \mu\left(A_{f}\right)=0$.

LEMMA 3.4. For each idempotent $p \in S, \theta \mu\left(K_{p}\right)=0$.

Proof. If $\theta \mu\left(K_{p}\right)=\delta>0$ for some idempotent $p \in S$, then there is topology $\tau \epsilon \mathscr{T}\left(G\left(\tau_{0}\right)\right)$ such that $N_{p}=L^{1 / 2}(G(\tau))([10])$. Let $n$ be an integer with $\frac{1}{2^{n-1}}<\delta$. Since $\left\|\mu^{\alpha}\right\|=\frac{1}{2^{n}}$ for all $\alpha \in \Lambda_{n}$ and $\mu=\sum_{a \in \Lambda_{n}} \mu^{\alpha}$, there exists at least three distinct elements $\alpha_{1}, \alpha_{2}, \alpha_{3}$ of $\Lambda_{n}$ such that $\mu^{\alpha_{i}}(i=1,2,3)$ are not singular to $L^{1 / 2}(G(\tau))$. Let $\omega_{i}$ be non-zero positive measure of $L^{1 / 2}(G(\tau))$, with $\omega_{i} \ll \mu^{\alpha_{i}}(i=1,2,3)$. Then, for some integer $k,\left(\omega_{1} * \omega_{2}\right)^{k}$ is not singular to $L^{1}(G(\tau))\left([10]\right.$, p. 112). On the other hand, from $\left(\omega_{1} * \omega_{2}\right)^{k}$ $\ll\left(\mu^{\alpha_{1}} * \mu^{\alpha_{2}}\right)^{k} \ll\left(m_{\alpha_{1} \wedge a_{2}}\right)^{k}=m_{a_{1} \wedge a_{2}}$ it follows that $\left(\omega_{1} * \omega_{2}\right)^{k} \in L^{1}\left(G\left(\tau_{\alpha_{1} \wedge \alpha_{2}}\right)\right.$, where $\tau_{a_{1} \wedge a_{2}}$ is the weakest locally compact group topology on $G$ such that $G_{\alpha_{1} \wedge a_{2}}$ is open. Since $L^{1}(G(\tau)) \cap L^{1}\left(G\left(\tau_{a_{1} \wedge a_{2}}\right)\right) \neq\{0\}, \quad \tau=\tau_{a_{1} \wedge a_{2}}$.

On the other hand, since $\left(\omega_{1} * \omega_{2} * \omega_{3}\right)^{k}$ is not singular to $L^{1}(G(\tau))$ and $\left(\omega_{1} * \omega_{2} * \omega_{3}\right)^{k} \ll m_{\alpha_{1} \wedge a_{2} \wedge a_{3}}$, we have $\tau=\tau_{\alpha_{1} \wedge \alpha_{2} \wedge \alpha_{3}}$. But, from $H_{\alpha_{3}}$ 
being an uncountable compact group, it follows that $\tau_{\alpha_{1} \wedge \alpha_{2}} \neq \tau_{\alpha_{1} \wedge \alpha_{2} \wedge \alpha_{3}}$. Thus we have reached a contradiction.

Since $\Lambda$ is a countable set, we can get the following theorem.

THEOREM 3.5. If $G\left(\tau_{0}\right)$ is the complete direct sum of an infinite family of infinite compact abetian groups, then $M_{\mathcal{K}}\left(G\left(\tau_{0}\right)\right)$ is non-trivial.

COROLLARY. If $G\left(\tau_{0}\right)$ is a compact group such that the dual group $\hat{G}\left(\tau_{0}\right)$ of $G\left(\tau_{0}\right)$ has an infinite independent set $P$, then $M_{K}\left(G\left(\tau_{0}\right)\right) \neq\{0\}$.

Proof. Let $\left\{P_{n}\right\}_{n=1}^{\infty}$ be a disjoint collection of infinite subsets of $\hat{G}\left(\tau_{0}\right)$, with $P=\bigcup^{\infty} P_{n}$. Let $H$ be the annihilator of the group generated. by $P$. Then $G\left(\tau_{0}\right) / H$ is the complete direct sum of a countable family $\left\{H_{n}\right\}_{n=1}^{\infty}$ of infinite compact groups, where $H_{n}$ are dual groups of infinite discrete groups generated by $P_{n}\left([7]\right.$, p. 37). Let $S_{1}$ and $S_{2}$ be the structure semigroups of $M\left(G\left(\tau_{0}\right)\right)$ and $M\left(G\left(\tau_{0}\right) / H\right)$, respectively. Let $\theta_{1}$ and $\theta_{2}$ be the homomorphisms of $M\left(G\left(\tau_{0}\right)\right)$ into $M\left(S_{1}\right)$ and of $M\left(G\left(\tau_{0}\right) / H\right)$ into $M\left(S_{2}\right)$ with properties $(1)-(4)$ in this section, respectively. By Theorem 3.5, $M_{K}\left(G\left(\tau_{0}\right) / H\right)$ is non-trivial.

Let us denote by $\varphi$ the canonical homomorphism of $G\left(\tau_{0}\right)$ to $G\left(\tau_{0}\right) / H$ and by $\Phi$ the induced Banach algebra homomorphism from $M\left(G\left(\tau_{0}\right)\right.$ onto $M\left(G\left(\tau_{0}\right) / H\right)([7]$, p. 54). There exists a non-zero positive measure $\mu \in M\left(G\left(\tau_{0}\right)\right)$ such that $\Phi_{\mu}$ is a measure of $M_{K}\left(G\left(\tau_{0}\right) / H\right)$. Since $\Phi\left(m_{H}\right)$ is the unit of $M\left(G\left(\tau_{0}\right) / H\right)$, we may assume that $\mu * m_{H L}=\mu$.

At first we shall show for any $f \in \hat{S_{1}}$

$$
|f|^{2}(s)=|f|(s) \quad \text { for } s \in S_{1}, \theta_{1} \mu \text { a.e., }
$$

if $|f|\left(m_{H}\right)=0$, then

$$
\int|f| d \theta_{1} \mu=|f|(\mu)=|f|\left(\mu * m_{H}\right)=|f|(\mu)|f|\left(m_{H}\right)=0,
$$

so that $|f|(s)=0 \quad \theta_{1} \mu$ a.e. If $|f|\left(m_{I I}\right) \neq 0$, then $|f|(s)=1$ for $s \in S_{1}$ $\theta_{1} m_{H}$ a.e. ([10], p. 112). Thus, by Lemma 3.2 in [1], there is a positive semicharacter $g \in \hat{S_{2}}$ such that

$$
|f|(\nu)=g(\Phi \nu) \quad \text { for each } \nu \in M\left(G\left(\tau_{0}\right)\right) .
$$

Put $A_{f}=\left\{s \in S_{1}: 0<|f|(s)<1\right\}$. If $\theta_{x} \mu\left(A_{f}\right)>0$, then there is a non-zero positive measure $\omega \in M\left(G\left(\tau_{0}\right)\right)$ such that $\theta_{1} \omega$ is the restriction measure to $A_{f}$ of. $\theta_{1} \mu$ (cf. [9]). From the inequality

$$
\int g d \theta_{2} \Phi \omega=\int|f| d \theta_{1} \omega=\int_{A_{f}}|f|(s) d \theta_{1} \omega(s)<\left\|\theta_{1} \omega\right\|=\|\Phi \omega\|,
$$

it follows that $\theta_{2} \Phi_{\omega}\left(\left\{s \in S_{2}: g(s)<1\right\}\right)>0$. Since $\theta_{2} \Phi_{\omega} \ll \theta_{2} \Phi \mu$, we have $\Phi_{2} \omega \in M_{K}\left(G\left(\tau_{0}\right) / H\right)$, so that $\theta_{2} \Phi_{\omega}\left(\left\{s \in S_{2}: g(s)=0\right\}\right)>0$. Let $\nu$ be the measure on $G\left(\tau_{0}\right) / H$ such that $\theta_{2} \nu$ is the restriction to $\left\{s \in S_{2}: g(s)=0\right\}$ of $\Phi \omega$, then $\nu$ is a non-zero positive measure and $g(v)=0$. Let $\nu^{\prime}$ be a nonzero positive measure on $G\left(\tau_{0}\right)$ such that $\nu^{\prime} \ll \omega$ and $\Phi_{\nu^{\prime}}=\nu$. From $\nu^{\prime} \ll \omega$, it follows that $\theta_{1} \nu^{\prime} \ll \theta \omega$, thus

$$
g(v)=g\left(\Phi \nu^{\prime}\right)=|f|\left(\nu^{\prime}\right)=\int_{A_{f}}|f| d \theta_{1} \nu^{\prime}>0 .
$$

This is a contradiction.

Finally, we shall show $\mu \perp L^{1 / 2}(G(\tau))$ for every $\tau \in \mathscr{T}\left(G\left(\tau_{0}\right)\right)$. It is enough to show $\Phi\left(L^{1 / 2}(G(\tau))\right) \subset L^{1 / 2}(G(\tau) / H)$. Let $h$ be any multiplicative linear functional of $M(G(\tau) / H)$ such that $h(\nu)=0$ for every $\nu \in L^{1}(G(\tau) / H)$. Since $\Phi\left(L^{1}(G(\tau))\right)=L^{1}(G(\tau) / H)([7]$, p. 55$), h \circ \Phi$ is a multiplicative linear functional of $M(G(\tau))$ such that $h \circ \Phi\left(L^{1}(G(\tau))\right)=0$. Thus $h(\Phi \omega)=h \circ \Phi(\omega)$ $=0$ for every $\omega \in L^{1 / 2}(G(\tau))$. This shows that $\Phi\left(L^{1 / 2}(G(\tau))\right) \subset L^{1 / 2}(G(\tau) / H)$.

\section{References}

[1] W. J. Bailey, G. Brown and W. Moran, Spectra of independent power measures, Prof. Camb. Phil. Soc. 72 (1972), pp. 27-35.

[2] I.M. Gelfand, D.A. Raikov and G.E. Shilov, Commutative Normed Rings, New York 1964.

[3] C. C. Graham, Compact independent sets and Haar measure, Proc. Amer. Math. Soc. 36 (1972), pp. $578-582$.

[4] J. Inoue, Some closed subalgebras of measure algebras and a generalization of P. J. Cohen's theorem, J. Math. Soc. Japan 23 (1971), pp. 278-294.

[5] K. Izuchi, On a problem of J.L. Taylor, preprint.

[6] - and T. Shimizu, Topologies on groups and a certain L-ideals of measure algebras, Tôhoku Math. J. 25 (1973), pp. 53-60.

[7] W. Rudin, Fourier analysis on groups, New York 1962.

[8] K. Saka, A note on subalgebra of measure algebra vanishing on non-symmetria homomorphisms, Tôhoku Math. J. 25 (1973), pp. 333-338.

[9] J.L. Taylor, The structure of convolution on measure algebras, Trans. Amer. Math. Soc. 119 (1965), pp. 150-166.

[10] - L-subalgebras of $M(G)$, Trans. Amer. Math. Soc. 135 (1969), pp. 105-113.

[11] J.H. Williamson, Banach algebra elements with independent powers and theorems of Wiener-Pitt type, Function algebras, pp. 186-197, Chicago 1966.

[12] - Raikov systems and the pathology of $M(R)$, Studia Math. 31 (1968), pp. 399 $-409$

THE RESEARCH INSTITUTE OF APPLIED ELECTRICITY HOTKAIDO UNIVERSITY, SAPPORO, JAPAN 